\title{
Research Progress on High-Enthalpy and Hypersonic Flows
}

\author{
Z. Jiang, H. R. Yu, Z. B. Lin \\ LHD of Chinese Academy of Sciences, Institute of Mechanics, Beijing 100080, China \\ Email: zljiang@imech.ac.cn
}

\begin{abstract}
The research progress on high-enthalpy and hypersonic flows having been achieved in the Institute of Mechanics, Chinese Academy of Sciences, is reported in this paper. The paper consists of three main parts: The first part is on the techniques to develop advanced hypersonic test facilities, in which the detonation-driven shock-reflected tunnel and the detonation-driven shock-expanded tube are introduced. The shock tunnel can be used for generating hypersonic flows of a Mach number ranging from 10 to 20, and the expansion tube is applicable to simulate the flows with a speed of $7 \sim 10 \mathrm{~km} / \mathrm{s}$. The second part is dedicated to the shock tunnel nozzle flow diagnosis to examine properties of the hypersonic flows thus created. The third part is on experiments and numerical simulations. The experiments include measuring the aerodynamic pitching moment and heat transfer in hypersonic flows, and the numerical work reports nozzle flow simulations and flow non-equilibrium effects on the possible experiments that may be carried out on the above-mentioned hypersonic test facilities.
\end{abstract}

Key words: hypersonic flows, detonation driver, shock-reflected tunnels, shock-expanded tube, heat transfer.

\section{INTRODUCTION}

Hypersonics has been with us for many decades since the term "hypersonic" was first used in a paper by Tsien in $1946^{[1]}$ to describe a flow where the gas velocity is much greater than the ambient speed of sound. The velocity is so high that the vehicle travels at such a hypersonic speed could produce severe friction and intensive heat transfer. In supersonic fights, part of the kinetic energy of the vehicle's motion is absorbed by the air and carried away from the body through a process called viscous dissipation. However, the hypersonic vehicles create so high temperature that can actually cause chemical changes to occur in the fluid through which they fly. The most notable changes air undergoes as the temperature increases are dissociation and ionization. As the temperature increases, the assumptions about air properties are no longer valid and the vehicles are said to be traveling through a chemically-reacting boundary layer in which the air properties is varying from the vehicle nose to downstream. If the air properties change, namely density and heat transfer properties, the aerodynamic characteristics and heating properties of hypersonic vehicles can change drastically. Moreover, most of the hypersonic vehicles are intended to cruise at high altitudes where air density is low. In the low density flows, air can no longer be considered to be a continuum because the distance between individual particles of air becomes so great that each particle begins to affect the aerodynamic properties of the vehicles. Under such a situation, the common aerodynamic relations, like the Euler and Navier-Stokes equations, break down. Instead, aerodynamic properties must be analyzed using the kinetic theory. Some of the most important differences between the low density and continuous flows include the velocity slip that the viscous no-slip condition at the body surface fails since the friction is negligible for the low density flows and the flow velocity at the body surface is no longer zero, and the temperature slip that the gas temperature at the body surface being equal to the temperature of the body surface material fails.

Hypersonic flows behave quite different from supersonic ones, but there is no exact definition defining the start of the hypersonic flow regime. Possibilities are the Mach number at which supersonic linear theories fail and the specific heat ratio is no longer constant because of temperature effects on fluid properties. For blunt bodies, Mach 3 might be required to cause large disturbances to flows, and for the more highly-streamlined vehicles, Mach 5 might be the starting point of the hypersonic flow regime. As previously mentioned, the problems arising from the hypersonic flow research are not only related to fundamental fluid sciences, but also aerospace engineering. These problems may be referred 
as to the hypersonic "barrier" if one recalls the sonic "barrier" that had been overcome a few decades ago. The hypersonic "barrier" is actually the aerodynamic heat-induced barrier because it is the acrodynamic heat that results in fluid property changes, and leads to huge difficulties in development of hypersonic vehicles' TPS techniques and hypersonic propulsion.

The hypersonic vehicles are expected to travel at a speed ranging from Mach number 5 to 30 and at a altitude between $20 \mathrm{~km}$ and $100 \mathrm{~km}$. There are several primarily important problems associated with determining the aerodynamic environment of hypersonic vehicles and offering diverse challenges to both scientists and engineers. One of them is that the thermo-chemistry of the reacting gases generated around the hypersonic vehicles is difficult to match in ground-test facilities. Moreover, with exceptions for extremely brief test times, the high total enthalpy cannot be simulated in the ground-based test facilities without damaging the facility and/or the model. The second one is how to diagnose the aerodynamic the heat transfer, aerodynamic forces and moments acting on the vehicles in hypervelocity that is converted into increasing the temperature of the air and into endothermic reactions, such as dissociation and ionization of the air near the vehicle's surface. Another is modeling properly the aero-thermo-chemistry of the hypersonic flows including air dissociation, ionization, radiation and molecule vibrations. More particular problems had been identified by Bertin et al. in his review paper [2], and were listed as: the stagnation region, the boundary-layer transition, the viscous interactions and air-breathing hypersonic propulsion.

In this paper, the research progress on high-enthalpy and hypersonic flows in the Laboratory of High temperature Gas Dynamics (LHD) of Chinese Academy of Sciences in the Institute of Mechanics, is summarized in three parts. The first part is on the techniques to develop hypersonic test facilities, in which the detonation-driven shock-reflected tunnel (JF-10) and the detonation-driven shock-expanded tube (JF-16) are introduced. The shock tunnel was reported in the book edited by Lu and Marren [3], and is capable of simulating hypersonic flows with the velocity of $4 \sim 5 \mathrm{~km} / \mathrm{s}$ and total temperature as high as to $8000 \mathrm{~K}$. The expansion tube is applicable for generating the orbital speed flows of $7 \sim 9 \mathrm{~km} / \mathrm{s}$ with the total enthalpy higher than $30 \mathrm{MJ} / \mathrm{kg}$. The second part is dedicated to the nozzle flow diagnosis to examine the quality of the hypersonic flows thus created. Experimental data show that the improvement on $\mathrm{JF}-10$ shock tunnel is successful and the hypersonic flows generated are suitable for acrothermochemistry experiments. The last part is a brief introduction to some experiments and numerical simulations. The experiments include measuring the aerodynamic force acting on the blunt-nose cone and the aerodynamic heat transfer across surfaces of a two-dimensional symmetric wedge. The numerical work reports the JF-10 nozzle flow simulations and non-equilibrium effects on experiments that may be carried out on the JF-10 shock tunnel.

\section{DEVELOPMENT OF ADVANCED HYPERSONIC TEST FACILITIES}

In order to develop hypersonic vehicles, it is the most important to develop hypersonic test facilities for ground-based experiments. After more than 50 years study, high-enthalpy facilities suitable for studying aerothermochemistry are still based on shock tunnels [3]. Two kinds of high-enthalpy shock tunnels have been developed over the world. One of them is the free piston-driven shock tunnel, and the other is the detonation-driven ones. Several high-enthalpy shock tunnels had been built up and valuable experimental data are provided with the facilities for the present hypersonic study.

\section{Development of detonation-driven shock tunnels}

The detonation driver was first proposed by Bird in 1957, and a $13.3 \mathrm{~m}$ long detonation driver was built up by Yu et al. in 1981 and put into operation in 1983. Gronig et al. developed the one in Aachen, Germany in 1993 and their shock tunnel is operated in the backward mode. Later in 1994, the bigger one, named HYPULSE, was setup in GASL, USA [4], and could be also operated as a shock-expanded tube. These shock tunnels are operated either in the backward mode to achieve longer driving time at a relatively low enthalpy level, or in the forward mode to obtain the high enthalpy flow, but with a short test duration. To improve performances of detonation-driven shock tunnels in the forward mode to obtain the longer driving time, the forward detonation cavity driver (short for, the FDC driver) was proposed by Jiang [5] based on shock reflection concept. The detonation reflection in the FDC driver results in a strong upstream-traveling shock and a weak downstream-traveling one. The upstream-traveling shock elevated significantly the flow pressure behind the detonation front, which had decreased due to rarefaction waves. The downstream shock will catch up with the detonation front, the specially-designed FDC driver makes this shock so weak that an overdriven detonation could be avoided. After .Jiang's work, the JF-10 shock tunnel was re-built up with the new FDC driver in 
2003 , and its performance test was completed recently.

\section{Setup of the JF-10 shock tunnels}

The improved and original detonation-driven high enthalpy shock tunnels are schematically shown in Figure 1. The improved JF-10 shock tunnel, as shown in Figure 1b, consists of three main parts: the FDC driver is about $6.2 \mathrm{~m}$ in length; the driven section is $12.5 \mathrm{~m}$ long: and the conical nozzle with a $500 \mathrm{~mm}$ diameter exit. The original JF-10 has the same configuration, but its driver section is $10 \mathrm{~m}$ long, and about $4 \mathrm{~m}$ longer that the FDC driver. The shorter driver is effective in reduction of tunncl operation cost and nozzle throat ablation.

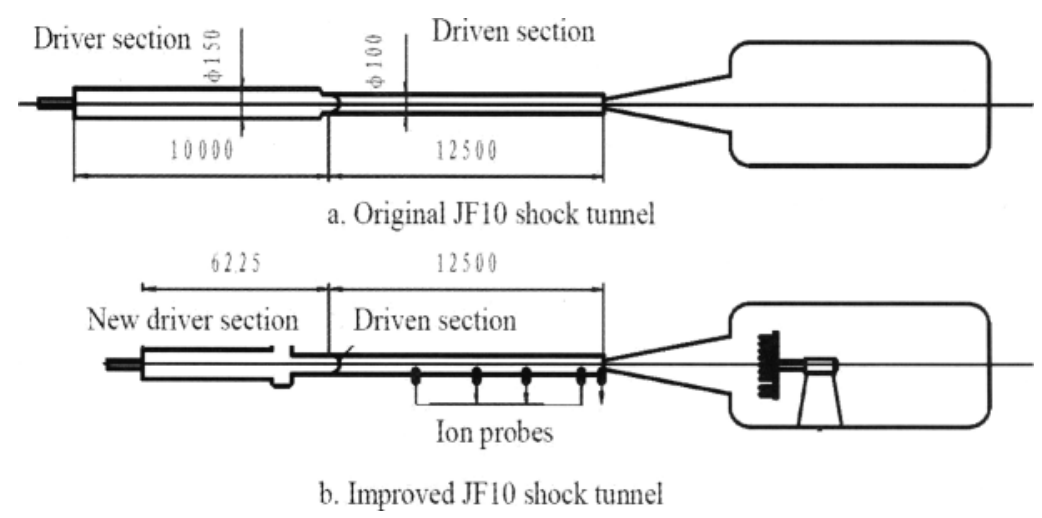

Figure 1: Schematic of the improved and original detonation-driven high enthalpy shockreflected tunnels

\section{Pressure variations at the end of the driven sections}

Figure 2 shows the pressure variation measured at the end of the driven section, and this pressure variation acts as the measure of the reservoir pressure during the nozzle working duration. From this figure, it is observable that the pressure level maintains approximately constant for more than $6 \mathrm{~ms}$ after the incident shock reflection By comparing the result with the 3 ms test time achieved with the HEIST in Kakuda, Japan, the performance of the improved JF-10 shock tunnel is very promising. Figure 3 shows the Pitot pressure variation measured in the nozzle flow of the improved JF-10 shock tunnel and the pressure profile looks similar to the pressure variation shown in Figure 2. The nozzle

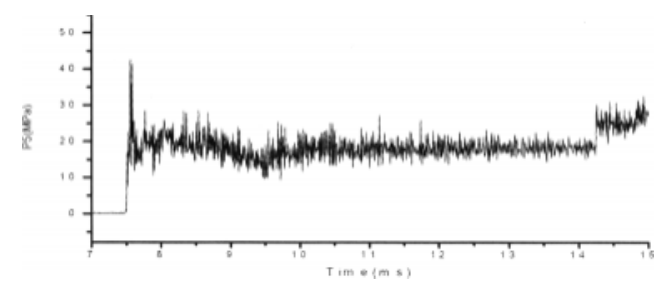

Figure 2: Pressure variations measured at the end of the driven section in the improved JF-10 shock tunnel

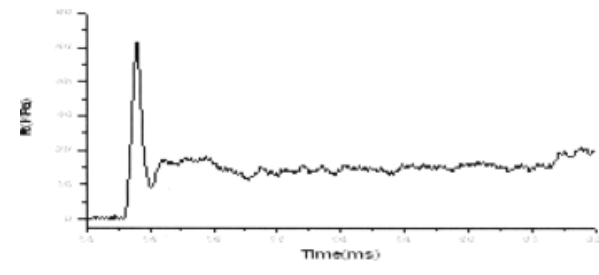

Figure 3: Pitot pressure variations measured in the nozzle flow of the improved JF-10 shock tunnel 
flow appears more stable than the reservoir gas state since the perturbations observable in the driven section die out quickly during the nozzle flow expansion. The similarity showed in Figures 2 and 3 demonstrates the experiment reliability.

\section{Detonation-driven shock-expanded tube}

The detonation-driven shock-expanded tube (JF-16) was setup in the LHD of Chinese Academy of Sciences in 2006 and its performance tests were completed recently. The tube performance was examined by measuring the incident shock speed and the wall static pressures at its expansion-acceleration section. By applying the incident shock speed, the velocity of hypersonic test flows was calculated with Mirels' theory [6] and test flow thermodynamic parameters were predicted with Gaseq software. The performance study shows that the velocity of above $7000 \mathrm{~m} / \mathrm{s}$, an test duration between and the total enthalpy high up to $30 \mathrm{MJ} / \mathrm{kg}$ have been achieved by using the JF-16 shock-expanded tube of 16.35 $m$ length. Wall static pressure histories at measurement point $\mathrm{B}$ and $\mathrm{C}$ at the expansion-acceleration section are shown in Figure 4. Point $\mathrm{C}$ is located more further downstream than point $\mathrm{B}$, and both results demonstrated a $50 \mu \mathrm{s}$ stable test duration.
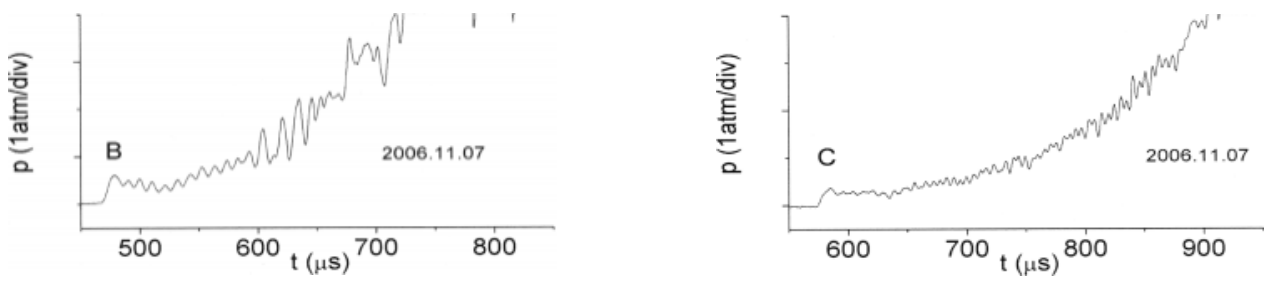

Figure 4: Wall static pressure histories at two measurement points at the expansion-acceleration section

\section{NOZZLE FLOW DIAGNOSIS}

\section{Nozzle flow uniformity}

To examine hypersonic test flow uniformity, five measurement points in the JF-10 nozzle flow are arranged along the nozzle centerline and the zero point is defined at the nozzle exit. The position distances are denoted for each measurement point with (a) $Z=-50 \mathrm{~mm}$; (b) $Z=100 \mathrm{~mm}$; (c) $Z=$ $265 \mathrm{~mm}$; (d) $Z=400 \mathrm{~mm}$ and (e) $Z=600 \mathrm{~mm}$, respectively. Measurements both in vertical and level directions are carried out at each measurement point, and the Pitot pressure profiles at point (b) and (d) are plotted together in Figure 5. It is observable that the test flow in the centre area appears quite uniform if evaluated from a view point of pressure variations, and actually, this uniform flow area is found to be approximately $700 \mathrm{~mm}$ in length and $400 \mathrm{~mm}$ in diameter. Although the pressure uniformity does not confirm the hypersonic flow uniformity, the results are quite promising and very encouraging. We are now working more actively to examine the more properties of the hypersonic test flow in an aerothermodynamics view point.
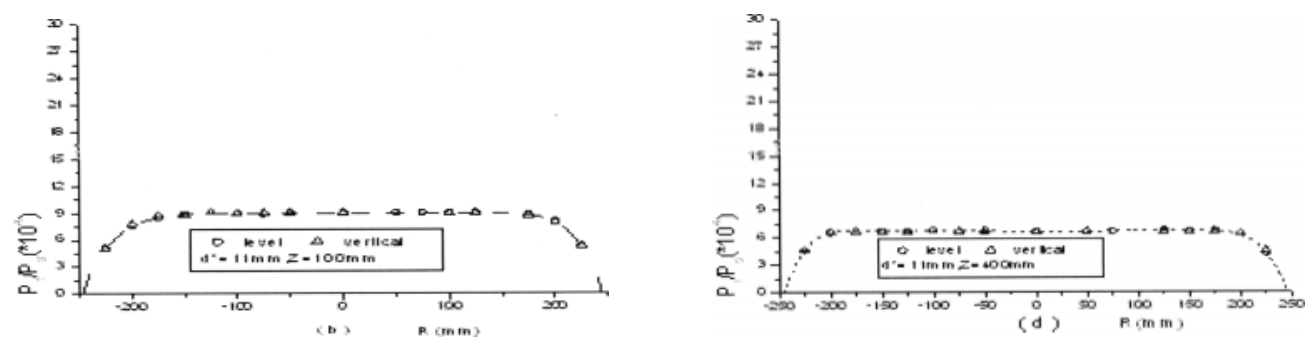

Figure 5: Point pressure profiles recorded at two measurement points in hypersonic nozzle flows

\section{The incident shock decay in the JF-10 driven section}

The incident shock decay in the driven section is a very important parameter for measuring hypersonic test facility performances and the test flow quality is affected significantly. For efficient comparison, 
three test cases are selected and the relevant test conditions are listed in Table 1. Cases A and B are carried out in the improved JF-10 shock tunnel, and case C is done in the original JF-10. Cases A and $\mathrm{B}$ are used to examine the incident shock decay at different initial conditions but the total flow enthalpy for the three cases are kept to be approximately the same. In these experiments, the incident shock speed is measured with several ion probes which are distributed along the driven section with equal intervals.

Table 1. Test conditions for investigation on the incident shock decay in the driven section

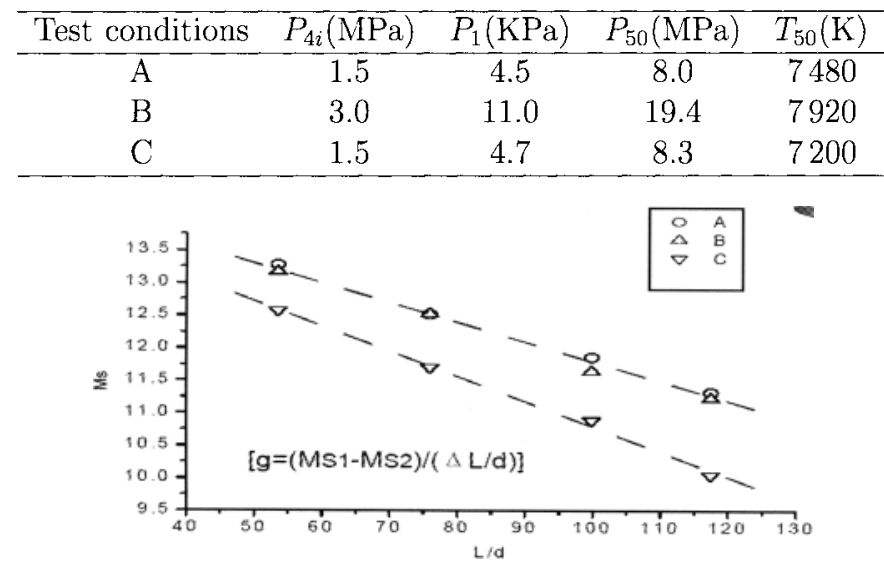

Figure 6: Incident shock wave decay along the driven section in three test cases

The incident shock decay rates along the driven section are presented in Fig. 6 and expressed with the Mach number drop per diameter. By comparing case A and B with case $\mathrm{C}$, it could be seen that much stronger incident shock could be driven out with the FDC driver and the Mach number could be $10 \%$ higher at the same initial condition. The decay rate is $3.04 \%$ in case A and $3.4 \%$ in case B, which means that the stronger shock will decay more, but the initial pressure rate in the driver and driven sections has no significant effect on the incident shock decay at the same total enthalpy. The incident shock decays faster in the original JF-10 than in the improved one, and therefore, the FDC driver has much better performance.

\section{EXPERIMENTAL AND NUMERICAL RESEARCHES}

\section{Measurement on pitching moments}

Because of the high total temperature, the test duration provided with hypersonic test facilities is extremely short and usually of a few milliseconds. This imposes a challenge for measuring aerodynamic force coefficients with traditional balance techniques. A three-component stress balance system was designed, fabricated and tested in the JF-10 shock tunnel to measure the drag, lift and pitching moments for a $30^{\circ}$ apex angle blunt cone within a $5 \mathrm{~ms}$ test time. The feasibility study is presented in Figure 7 in which the Point pressure profile in the nozzle flow is given in Figure $7 \mathrm{a}$ and the balance

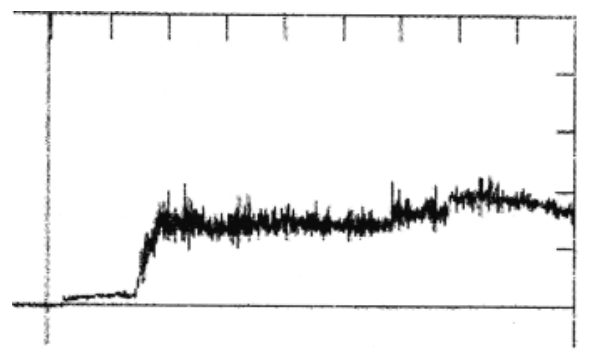

(a) Point pressure in the JF-10 nozzle flow

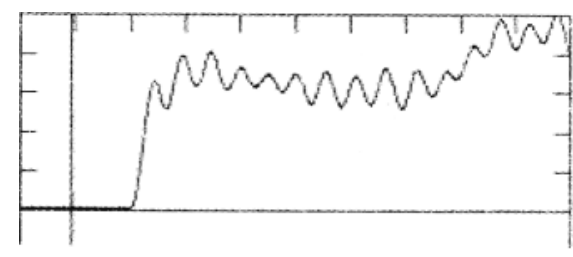

(b) Balance signs

Figure 7: Comparison of balance signs with the Point pressure profile in the JF-10 nozzle flows 
sign in Figure $7 \mathrm{~b}$. The agreement between the two results demonstrated that the balance has a good amplitude response. The balance amplitude response is actually within only one millisecond, so that the aerodynamic force experiments can be carried out on the JF-10 shock tunnel with the three-component stress balance system.

From flight tests, it was well known that the pitching moment during the re-entry of the Shuttle Orbiter was outside of the expected variations predicated with conventional hypersonic wind tunnels, causing body-flap trim problems. In order to demonstrate the real gas effect, how to setup a comparison base for hypersonic experiments is a key problem. Actually, it is meaningless to compare high enthalpy shock tunnel experiments with the conventional wind tunnels at the same nominal Mach number. It is because the gas expands from nominal total temperatures in the stagnation chamber of the wind tunnels to very low static temperatures in the test section, hypersonic Mach numbers are achieved through relatively low speeds of sound. Thus, for tests conducted in such the wind tunncls, Mach number is very large because the free-stream speed of sound becomes very small. As a result, the fluid temperatures in the flow fields around the models tested remain far below the levels that would damage the wind tunnel or the model. For flight tests, Mach number is very large because the freestream velocity is very high while the free-stream thermodynamic state remains fixed. The gas flow slows down as it crosses the shock wave that envelopes hypersonic vehicles, producing extremely high temperatures in the shock layer. The kinetic energy of the air particles in the flow field is converted into increasing the temperature of the air and into endothermic reactions, such as dissociation and ionization of the air.

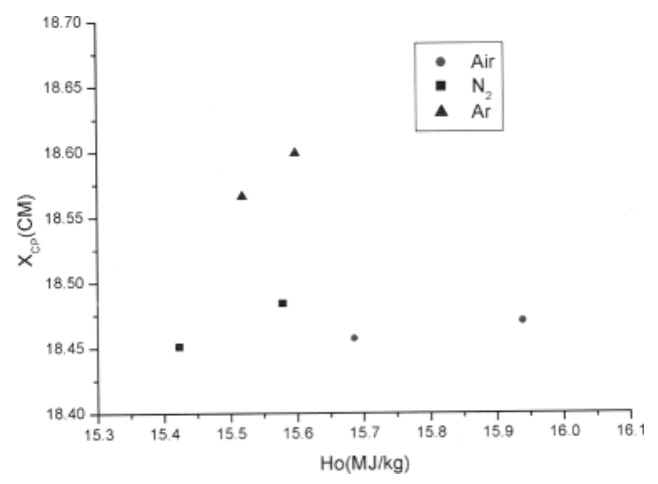

Figure 8: Comparison of the pitching moments in three cases of the air, Argon and Nitrogen

In order to examine the real gas effect, three test cases are so designed that the experimental data could be approximately comparable. For all the cases, the detonation wave created in the driver section has the same strength and the test gas in the driven section is compressed at the same pressure and temperature levels. The difference in the three cases is only the test gas. Argon is used for the first case to simulate the ideal gas, Nitrogen is chosen for the second case and the air for the last. At the total temperature of $7000 \mathrm{~K}$, estimated for air in the last case, three experiments were carried out and the results of the aerodynamic pitching moment are plotted in Figure 8. Around the total enthalpy of $15.6 \mathrm{MJ} / \mathrm{kg}$, the pitching moments appear dramatically different in the three cases. The air's data are higher than Nitrogen because the oxygen is easer to dissociate. The argon's data appear much higher than other two because that no dissociation occurs in Argon. The gas dissociation does not affect the flow pressure too much as one knew from the Mach number independence principle of hypersoni: flows, but changes the temperature significantly. The temperature change leads to the flow density change that is the reason why the pitching moments vary.

\section{The catalytic effect on heat transfer in hypersonic flows}

As a hypersonic vehicle is traveling through a chemically reacting boundary layer, the wall catalytic recombination will affect significantly the heat flux across the boundary layer to vehicle surfaces. In order to determine the catalytic effect on heat transfer rates at the wall surfaces with different physico-chemical properties at the same hypersonic flow condition that is difficult to match because of poor repeatability of high enthalpy test facilities, the special expcriment has to be designed and its efficiency needs to be validated. 
In order to solve the above-mentioned problem, a specially-designed test model is applied in the present test. It is a $10^{\circ}$ angle symmetric wedge having two surfaces: one surface is made of glass, serving as the non-catalytic surface, and the other is made of steel to simulate the catalytic wall. By setting the total temperature to be $7800 \mathrm{~K}$, two kinds of results can be achieved at the same shots and some experimental results are presented in Figure 9. In the figure, " $\triangle$ " denotes the catalytic wall result and "•" represents the data from the non-catalytic wall. The heat fluxes for both surfaces decrease as the measurement position moves downstream since the flow temperature decreases from the wedge nose to downstream. The hear flux of the non-catalytic wall is lower than the catalytic wall because the wall catalytic recombination is an exothermic reaction that will rise the flow temperature. The discrepancy between the two results is getting smaller and smaller because the gas dissociation is getting less and less intensive as the gas flow moves downstream. The catalytic effect is demonstrated with this experiment and the experimental results thus obtained must be more reliable due to the same free-stream condition.

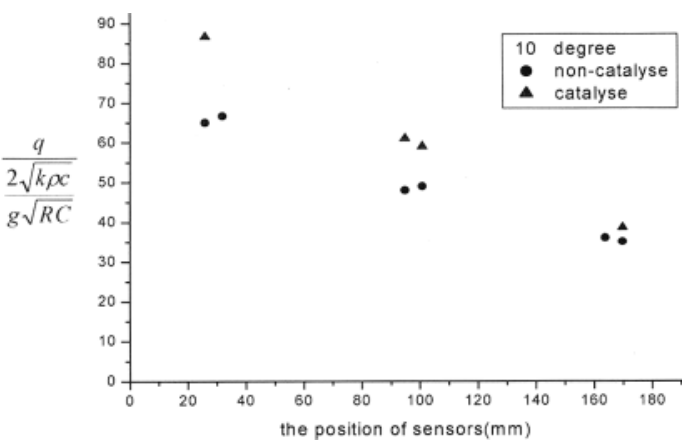

Figure 9: Comparison of the heat flux across a chemically-reacting boundary layer to noncatalytic and fully catalytic walls of a two-dimensional symmetric blunt wedge

\section{Nozzle flow equilibrium study}

For shock-reflected tunnels, the stagnation temperature is so high that the quickly-expanding test gas could be in a non-equilibrium state that will behave different from the one in flight tests. Such the difference may induce some errors in experimental data of ground tests and its effect has to be estimated.

Numerical simulations are applied to investigate the problem and the JF-10 nozzle is accepted as a test case. The conical nozzle of the JF-10 has a $11 \mathrm{~mm}$ diameter throat and $500 \mathrm{~mm}$ diameter exit, and its conc angle is $7^{\circ} 7^{\prime}$. Assuming the $19.6 \mathrm{MPa}$ total pressure and $7920 \mathrm{~K}$ total temperature, numerical simulations are carried out and its results are presented in Figure 10. The results show that the nozzle flow is in a thermo-equilibrium state at the beginning of flow expansionand then, becomes non-

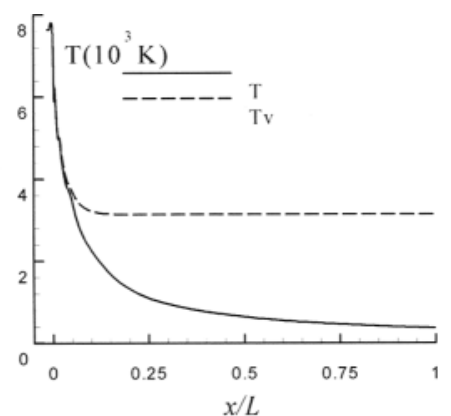

((a) The vibration and translation temperature

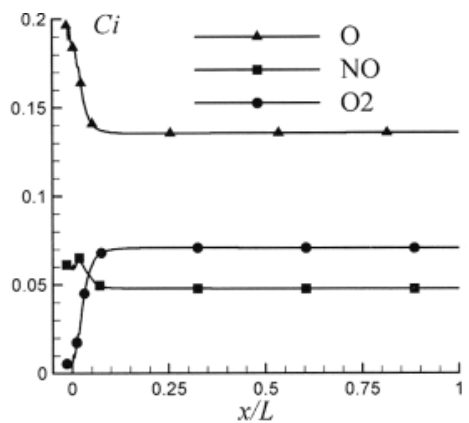

(b) Mass fraction distributions of $\mathrm{O}_{2} \mathrm{O}_{2}$ and $\mathrm{NO}$

Figure 10: Numerical results along the axis-symmetry of the JF-10 nozzle, showing thermodynamic equilibrium properties of the hypersonic nozzle flow 
equilibrium as the gas flow is expanding quickly, and finally had been frozen after $x / L=0.15$. Diverging of the vibration and translation temperatures, as shown in Figure 10a, indicates the nonequilibrium state, and the constant mass fraction distributions of $\mathrm{O}_{2} \mathrm{O}_{2}$ and $\mathrm{NO}$, as shown in Figure $10 \mathrm{~b}$, imply frozen chemical reactions. Further numerical simulations demonstrated that such the nonequilibrium test flow may induces a 3\% difference in the aerodynamic force measurement data by comparing it with the ideal equilibrium flow at the same total temperature and pressure for a blunt cone test model.

\section{CONCLUSIONS}

The research progress on high enthalpy and hypersonic flows is summarized as follows: The improvement of the JF-10 shock tunnel with a FDC driver was demonstrated to be very successful and the stronger incident shock could be generated with a lower decay rate at the same initial conditions. The test duration was extended to six milliseconds even the new driver is $40 \%$ shorter than the original one. The uniform hypersonic flow area is about $700 \mathrm{~mm}$ in length and $400 \mathrm{~mm}$ in diameter. Two experimental methods proposed for acrodynamic force and heating measurements were demonstrated to be acceptable, with which the real gas effects can be examined and experimental data will be more reliable. The nozzle flow may be in a non-equilibrium state in some extent, but the discrepancies induced by such the test flow falls in the level acceptable for engineering applications.

Our research progress shows that the "real gas effect" in hypersonic flow does change dramatically the aerodynamic characteristics of hypersonic vehicles and the developed hypersonic test facilities are suitable for the ground-based experiments of hypersonic flows. However, to gain more reliable experimental data, it is the most important to develop new diagnosis techniques and propose some creative methods for hypersonic experiments in future.

\section{Acknowledgements}

The support of the National Natural Science Foundation of China under projects of 90205027 and 10632090 is gratefully acknowledged.

\section{REFERENCES}

1. Tsien HS. Similarity laws of hypersonic flows. J. Math and Phys, 1946; 25: 247-251

2. Bertin JJ, Cummings RM. Fifty years of hypersonics: where we've been, where we're going. Progress in Aerospace Sciences, 2003; 39: 511-536

3. Lu FK, Marren DE. Advanced Hypersonic Test Facility. Progress on Astronautics and Aeronautics. 2002: 198

4. Wilson GJ, Sussman MA, Bogdanoff DW. A study of combustion and detonation drivers for high enthalpy expansion tubes and shock tubes. NASA CDTP-20000, 1995

5. Jiang Z, Zhao W, Wang C. Forward-running detonation drivers for high-enthalpy shock tunnels. AlAA Journal, 2002; 40: 2009-2016

6. Mirels H. Test time in low-pressure shock tubes. Physics of Fluids, 1963; 6(9): 1201-1214 